\title{
Lung function, COPD and Alternative Healthy Eating Index in US adults
}

\author{
Kirstie Ducharme-Smith ${ }^{1}$, Gustavo Mora-Garcia ${ }^{2}$, Francisca de Castro Mendes ${ }^{3}$, Maria Stephany Ruiz-Diaz ${ }^{4}$, \\ Andre Moreira ${ }^{5}$, Rodrigo Villegas ${ }^{6}$ and Vanessa Garcia-Larsen $\mathbb{D}^{3}$
}

${ }^{1}$ Dept of International Health, Johns Hopkins Bloomberg School of Public Health, Baltimore, MD, USA. ${ }^{2}$ Dept of Family Medicine and Public Health, Faculty of Medicine, Universidad de Cartagena, Cartagena de Indias, Colombia. ${ }^{3}$ Program in Human Nutrition, Dept of International Health, The Johns Hopkins Bloomberg School of Public Health, Baltimore, MD, USA. ${ }^{4}$ Center for Innovation and Research in Diabetes and Metabolism (INNOVATID), Cartagena de Indias, Colombia. ${ }^{5}$ Serviço de Imunologia Básica e Clínica, Departamento de Patologia, Faculdade de Medicina; EPI Unit - Instituto de Saúde Pública, Universidade do Porto, Porto, Portugal. ${ }^{6}$ Biostatistics Unit, School of Public Health, University of Chile, Santiago, Chile.

Corresponding author: Vanessa Garcia-Larsen (vgla@jhu.edu)

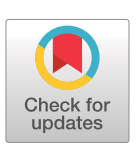

Copyright $\odot$ The authors 2021

This version is distributed under the terms of the Creative Commons Attribution Non-Commercial Licence 4.0. For commercial reproduction rights and permissions contact permissions@ersnet.org

This article has supplementary material available from openres.ersjournals.com

Received: 9 Dec 2020 Accepted: 25 July 2021

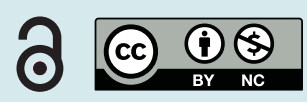

Shareable abstract (@ERSpublications)

In this nationally representative population-based study of US adults, diet quality measured by the AHEI-2010 was low. Better diet quality was associated with better lung function, and with lower prevalence of spirometric restriction. https://bit.ly/3you2Tf

Cite this article as: Ducharme-Smith K, Mora-Garcia G, de Castro Mendes F, et al. Lung function, COPD and Alternative Healthy Eating Index in US adults. ERJ Open Res 2021; 7: 00927-2020 [DOI: 10.1183/ 23120541.00927-2020].

\section{Abstract}

Background There is a large burden of COPD in the US. The purpose of this study was to investigate the association between diet quality with lung function, spirometric restriction and spirometrically defined COPD in a nationally representative sample of US adults.

Methods Adults (19-70 years of age) from the National Health and Nutrition Examination Survey 20072012 cycles were included ( $\mathrm{n}=10428)$. Diet quality was determined using the Alternative Healthy Eating Index (AHEI-2010). Pre-bronchodilator measurements of forced expiratory volume in $1 \mathrm{~s}\left(\mathrm{FEV}_{1}\right)$, forced vital capacity (FVC) and the $\mathrm{FEV}_{1} / \mathrm{FVC}$ were described. Calibrated lower limit of normal (LLN) estimates were derived to determine prevalence of spirometric restriction (FVC $<\mathrm{LLN}$ ) and $\mathrm{COPD}\left(\mathrm{FEV} \mathrm{F}_{1} / \mathrm{FVC}\right.$ ratio $<$ LLN). Population-weighted linear and logistic regression models were used to investigate the association of AHEI-2010 and respiratory outcomes.

Results The mean \pm SD AHEI was $45.3 \pm 12.2$, equivalent to meeting $41 \%$ of the daily recommendations for optimal diet quality. Those in the highest quartile of AHEI had better $\mathrm{FEV}_{1}$ (adjusted (a) $\beta$ 47.92, 95\% CI 2.27-93.57) and FVC (a 3 80.23, 95\% CI 34.03-126.42; p-value interaction (*) of AHEI and smoking $>0.05$ ) compared to those in quartile 1. Higher AHEI was also associated with lower odds of spirometric restriction (OR 0.23, 95\% CI 0.08-0.67; p-value AHEI*ethnicity >0.05).

Conclusions Diet quality was independently associated with better $\mathrm{FEV}_{1}$ and FVC and with lower odds of spirometric restriction. These findings highlight the need for research to further elucidate the possible beneficial role of diet in the preservation of lung function.

\section{Introduction}

Poor lung function and COPD are major threats to public health in the US. In 2016, COPD was the third leading cause of disability adjusted life-years [1]. In 2014 and 2015, 6.4\% of adults in the US aged 40 years or older had been diagnosed with COPD, and these individuals were more likely to have heart disease, cancer and diabetes [2]. The prevalence of COPD is higher among individuals with lower income and on public insurance [2], placing around $11.8 \%$ of US adults with poverty (in 2018) at increased risk for the development of COPD [3].

The major risk factors for poor lung function are older age and having a history of smoking; however, this only accounts for around half of the cases of COPD [4]. Increasing epidemiological evidence has 
demonstrated that dietary intake can impact lung health [5, 6]. Evidence from cohort studies among European adults has demonstrated that higher fruit intake is associated with slower lung function decline [5-8]. Current evidence in US adults suggests that consumption of fruit and vegetables is associated with reduced incidence of COPD and that higher fibre intake is associated with better lung function [9, 10]. In addition to individual dietary components, overall diet quality has been associated with lung health in US adults. The Alternative Healthy Eating Index (AHEI-2010), an 11-component index that measures diet quality (higher score=better diet quality), was associated with decreased incidence of self-reported or newly diagnosed COPD in women from the Nurses Health Study from 1984 to 2000 [11]. To our knowledge, no studies in the US have investigated the association between AHEI-2010 with impaired lung function and spirometrically defined COPD in a nationally representative sample of the general population.

Therefore, the purpose of this study was to examine the association of diet quality as measured by the AHEI-2010 and lung function, spirometric restriction, and spirometrically defined COPD in US men and women who participated in the National Health and Nutrition Examination Survey (NHANES) 2007-2012.

Materials and methods

\section{Study sample}

The NHANES survey is an ongoing cross-sectional study carried out in representative samples of the US population [12]. Spirometry data were collected in NHANES cycles 2007-2008, 2009-2010 and 2011-2012. Individuals between 19 and 70 years of age who completed at least one 24-hour dietary recall and underwent the spirometry examination in the NHANES 2007-2012 cycles were included in the current study.

\section{Lung function and COPD measures}

Presence of asthma (ever, current) and bronchitis (ever, current) was collected via self-report from the Medical Conditions questionnaire [13]. Pre-bronchodilator measures of forced expiratory volume in $1 \mathrm{~s}$ $\left(\mathrm{FEV}_{1}\right)$ and forced vital capacity (FVC) were collected [14]. Measurements were carried out in the standing position; nose clips were worn to prevent air leaks and participants were instructed to take their deepest breath possible and then blow air into the mouthpiece out as hard and fast as possible for a minimum of $6 \mathrm{~s}$ of exhalation. Testing continued until participants were able to achieve a reproducible spirogram, or until a maximum of eight spirometry curves had been collected, or until the participant could not continue [14]. Quality of each valid spirometry measure was registered following the American Thoracic Society (ATS) collection standards. Only measurements that were graded A (exceeds ATS data collection standards) or B (meets ATS data collection standards) were included in the current analysis [15].

The European Respiratory Society Global Lung Function Initiative predictive equations were utilised to determine per cent predicted values for lung function measures based on age, height, sex and ethnicity [16]. Lower limit of normal (LLN) values were calculated to define spirometric restriction(FVC $<$ LLN) and $\mathrm{COPD}\left(\mathrm{FEV}_{1} / \mathrm{FVC}<\mathrm{LLN}\right)$ [17]. As pre-bronchodilator spirometry to derive norms for lung function reduces sensitivity compared to a post-bronchodilator gold standard, we adjusted these values by a constant to improve validity of the test, as recommended by KATO et al. [18].

\section{Diet quality}

The AHEI-2010 score was calculated using estimates of daily food and nutrient intake obtained either from one $(n=10428)$ or averaged over two repeated 24-hour recall questionnaires $(n=9224 ; 88 \%)$. The AHEI-2010 score is based on 11 components of dietary intake scored from 0 to 10 (worst to best) with total score ranging between 0 and 110 (non-adherence to perfect adherence). Scores increase with higher intakes of fruit, vegetables, whole grains, nuts and legumes, omega-3 fatty acids, polyunsaturated fatty acids (PUFA) and moderate consumption of alcohol. Scores decrease with higher intakes of sugar-sweetened beverages (SSB), red/processed meat, trans-fat, sodium and heavy consumption of alcohol. Individual food components were scored following criteria by CHIUve et al. [19] from which the AHEI-2010 score was derived.

\section{Potential confounders}

Demographic information was collected via questionnaires, in the home, by trained interviewers using the Computer-Assisted Personal Interviewing (CAPI) system [20]. The demographic questionnaire included self-reported information on age, race, sex, language, education, income and physical activity.

Current cigarette use and recent tobacco use were obtained via self-report. This information and urinary cotinine were utilised to determine smoking status [21, 22]. Participants who indicated "I have never 
smoked, not even a puff" in response to the question "Cigarettes smoked in entire life" or who had a urinary cotinine value $<1 \mathrm{ng} \cdot \mathrm{mL}^{-1}$ were classified as never-smokers [22]. Those who reported any amount of time to the question "How long since quit smoking cigarettes" or who had a urinary cotinine value 1-10 ng $\cdot \mathrm{mL}^{-1}$ were classified as former smokers [22]. In addition, those who reported they "Did not know" to the previous question and also reported "Not at all" in response to the question "Do you now smoke cigarettes" were also classified as former smokers. Participants who responded "Every day" or "Some days" to the question "Do you now smoke cigarettes" or who had a urinary cotinine value $>10 \mathrm{ng} \cdot \mathrm{mL}^{-1}$ were classified as current smokers [22]. Physical activity data were collected via self-report. Participants were classified as "physically active” if they reported engaging in moderate physical activity for 150 min per week or vigorous physical activity for 75 min per week [23].

Anthropometric data, including height and weight, were measured in all survey participants [24]. Standing height was collected with a stadiometer and was recorded in centimetres. Weight was measured using a digital scale and was recorded in kilograms. Body mass index was calculated as weight (kilogrammes) over squared height (metres squared), and classification of weight status was determined as underweight for $<18.5 \mathrm{~kg} \cdot \mathrm{m}^{-2}$, normal for $18.5-24.9 \mathrm{~kg} \cdot \mathrm{m}^{-2}$, overweight for $25-29.9 \mathrm{~kg} \cdot \mathrm{m}^{-2}$ or obese for $\geqslant 30 \mathrm{~kg} \cdot \mathrm{m}^{-2}$.

\section{Analyses}

Continuous variables were examined to determine distribution of sample characteristics and presented as means with standard deviation. Non-normally distributed data were presented as medians with interquartile range. Categorical variables were presented as numbers with percentages. Participants were excluded from analyses if they had unrealistic reporting of caloric intake determined by kilocalorie/basal metabolic rate (BMR) ratio (kcal/BMR): ratio $<0.5$ th percentile or $>99.5$ th percentile [25]. Among the remaining sample, quartile AHEI-2010 scores were calculated based on the sample distribution within each NHANES cycle year. Demographic and dietary intake was summarised over the total sample and as stratified by quartile AHEI-2010 score. Dietary data are compared to the US Department of Agriculture 2015-2020 Dietary Guidelines for Americans to provide context to the results [26].

NHANES sample weights [27] were utilised to determine prevalence estimates of spirometric restriction and COPD. Population-weighted linear regression models were used to assess the association between quartile diet quality score and lung function measures (pre-bronchodilator $\mathrm{FEV}_{1}$, FVC and $\mathrm{FEV}_{1} / \mathrm{FVC}$ ratio) among the entire sample $(\mathrm{N}=10428)$ and in subgroups based on race/ethnicity or smoking status. The analyses were tested and met the assumption for linearity between the standard residuals and the predictor variables. Population-weighted logistic regression models were used to assess the association between quartile diet quality, $\mathrm{COPD}\left(\mathrm{FEV}_{1} / \mathrm{FVC}<\mathrm{LLN}\right)$ and spirometric restriction $(\mathrm{FVC}<\mathrm{LLN})$ among the entire sample ( $n=10428)$, and in subgroups based on race/ethnicity or smoking status. Potential confounders were explored between diet quality (per quartile increase) and COPD with the addition of individual AHEI-2010 components.

Regression models were adjusted for several confounding factors. Model 1 controlled for age (years), sex (male, female) and height (centimetres). Model 2 controlled for age (years), sex (male, female), height (centimetres), race/ethnicity (non-Hispanic white, non-Hispanic black, Hispanic, other), total energy intake (kilocalories), education (Khigh school, >high school, unknown), household income (USD 0-25000, $25000-55000, \geqslant 55000$ or unknown), body mass index (BMI) category (underweight, normal, overweight, obese), smoking status (nonsmoker, former smoker, current smoker) and whether the participant was physically active (yes, no). Interactions between AHEI and smoking, and AHEI and ethnicity were investigated for each outcome under study. Subgroup analyses by race/ethnicity and by smoking status were also carried out, adjusting for the covariates described in Model 2. Additional analyses adjusting individually by each AHEI food group and by dietary patterns (derived from principal component analysis) were also carried out. Statistical significance was identified as p-value $<0.05$. Stata 14 (StataCorp LP, College Station, TX, USA) was used for all analyses [28].

\section{Results}

There were 10428 individuals with high-quality spirometry measures who had plausible dietary intake (figure 1). The general characteristics of the participants included in the study as well as their outcome and dietary data are summarised in table 1 . The mean \pm SD age of participants was $42.9 \pm 14.7$ years; the majority of participants identified as non-Hispanic white $(n=4452,43 \%)$, spoke English $(n=9008,86 \%)$ and had graduated from high school $(n=5673,54 \%)$. Around half of participants had an annual household income <USD 55 000, and only 31\% ( $\mathrm{n}=3275)$ were considered physically active. Although half of the participants $(n=5006)$ were identified as never-smokers based on self-report and urinary cotinine values $<1 \mathrm{ng} \cdot \mathrm{mL}^{-1}$, around one-third of participants $(n=3292)$ were found to be current smokers based on self-report and urinary 
Total participants NHANES 2007-2012 $n=30442$

$\nabla$

Individuals between

19 and 70 years of age $\mathrm{n}=15284$

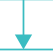

Individuals with at least one

24-hour recall $n=13904$

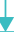

Individuals with valid dietary data (energy/BMR ratio 0.5-99.5th percentile) $n=13582$

Individuals with spirometry measures $n=12003$

Individuals with spirometry measures graded A (exceeds ATS data collection standards) or B (meets ATS data collection standards) $\mathrm{n}=10428$

FIGURE 1 Flow diagram of participants from the National Health and Nutrition Examination Survey (NHANES) included in the study. BMR: basal metabolic rate; ATS: American Thoracic Society.

cotinine values $>10 \mathrm{ng} \cdot \mathrm{mL}^{-1}$ (table 1 ). Statistically significant differences in demographic characteristics were found by quartile diet quality score ( $\mathrm{p}$-value $<0.001$ for all). Individuals with higher diet quality scores (Quartile 4) were more likely to be older, female, non-Hispanic white, educated, moderately active and nonsmokers compared to individuals with lower diet quality scores (Quartile 1).

On average, participants were overweight (BMI \pm SD 29.03 \pm 6.82 ), and $37 \%(n=3887)$ were classified as obese. Around $15 \%(\mathrm{n}=1501)$ of the study population reported having a history of asthma, and $8 \%$ $(n=782)$ reported current asthma. Only 5\% $(n=483)$ reported having a history of bronchitis, and $2 \%$ $(\mathrm{n}=205)$ reported current bronchitis. The mean $\pm \mathrm{SD}$ pre-bronchodilator $\mathrm{FEV}_{1}, \mathrm{FVC}$ and $\mathrm{FEV}_{1} / \mathrm{FVC}$ ratio were $3.2 \pm 0.9 \mathrm{~L}, 4.0 \pm 1.1 \mathrm{~L}$ and $79 \pm 8 \%$, respectively. Lung function measures were, on average, normal compared to the per cent predicted values for age, height, sex and ethnicity. Prevalence of spirometric restriction was $1.6 \%$ and COPD was $4.6 \%$. Statistically significant differences in physical measures were found by quartile diet quality score. Individuals with higher quality diet scores (Quartile 4) were less likely to be overweight/obese $(p<0.001)$, less likely to have self-reported asthma $(p<0.01)$ or bronchitis $(p<0.001)$, tended to have higher lung function measures $(p<0.001)$ and were less likely to have spirometric restriction $(\mathrm{p}=0.072)$ and COPD $(\mathrm{p}<0.001)$ than those with lower diet quality scores.

Demographic characteristics of study participants with spirometric restriction and COPD can be found in supplementary table S1. These participants tended to be older, have lower household income and be current smokers compared with the total sample. Individuals with spirometric restriction were more likely to be male and identify as non-Hispanic black, whereas individuals with COPD were more likely to be female and identify as non-Hispanic white compared with the total sample.

Participants had a mean (SD) total energy intake of of $2129( \pm 826) \mathrm{kcal}^{-}$day $^{-1}$, with carbohydrates being

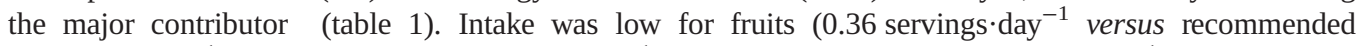
4 servings $\cdot$ day $\left.^{-1}\right)$, vegetables $\left(0.86\right.$ servings $\cdot$ day $^{-1}$ versus recommended 5 servings $\cdot$ day $\left.^{-1}\right)$, whole grains (6.4 $\mathrm{g} \cdot$ day $^{-1}$ versus recommended $75-90 \mathrm{~g} \cdot$ day $^{-1}$ based on sex) and PUFA (7.1\% of total calories versus recommended $10 \%$ of total calories) but high for SSB (1.21 servings $\cdot$ day $^{-1}$ versus 1 serving $\cdot$ day $^{-1}$ limit), 
TABLE 1 Characteristics of participants stratified by quartile Alternative Healthy Eating Index (AHEI-2010) score from National Health and Nutrition Examination Survey (NHANES) cycles 2007-2012 ( $n=10428)$

Quartile AHEI-2010 score"

\begin{tabular}{|c|c|c|c|c|c|}
\hline & \multirow[b]{2}{*}{ Total sample } & \multicolumn{4}{|c|}{ Quartile AHEI-2010 score" } \\
\hline & & Quartile 1 & Quartile 2 & Quartile 3 & Quartile 4 \\
\hline Subjects $\mathrm{n}$ & 10428 & 2608 & 2607 & 2608 & 2605 \\
\hline \multicolumn{6}{|l|}{ Demographics } \\
\hline Age years (mean $\pm S D$ ) & $42.9 \pm 14.7$ & $38.1 \pm 14.0$ & $41.0 \pm 14.3$ & $44.2 \pm 14.6$ & $48.2 \pm 13.9$ \\
\hline Male sex & $5123(49.1)$ & $1649(63.2)$ & $1336(51.3)$ & $1206(46.2)$ & $932(35.8)$ \\
\hline \multicolumn{6}{|l|}{ Race } \\
\hline Non-Hispanic white & $4452(42.7)$ & $1155(44.3)$ & $1065(40.9)$ & $1056(40.5)$ & $1176(45.1)$ \\
\hline Non-Hispanic black & $2185(21.0)$ & $665(25.5)$ & $581(22.3)$ & $520(19.9)$ & 419 (16.1) \\
\hline Hispanic & $2918(28.0)$ & $623(23.9)$ & $789(30.3)$ & $819(31.4)$ & $687(26.4)$ \\
\hline Other & $873(8.3)$ & $165(6.3)$ & $172(6.6)$ & $213(8.2)$ & $323(12.4)$ \\
\hline \multicolumn{6}{|l|}{ Language } \\
\hline English & $9008(86.4)$ & $2356(90.3)$ & $2220(85.2)$ & $2172(83.3)$ & $2260(86.8)$ \\
\hline Spanish & $1420(13.6)$ & $252(9.7)$ & $387(14.8)$ & $436(16.7)$ & $345(13.2)$ \\
\hline \multicolumn{6}{|l|}{ Education } \\
\hline$\leqslant$ High school & $183(1.8)$ & $83(3.2)$ & $57(2.2)$ & $32(1.2)$ & $11(0.4)$ \\
\hline >High school & $5673(54.4)$ & $1165(44.7)$ & $1322(50.7)$ & $1432(54.9)$ & $1754(67.3)$ \\
\hline Unknown & $4572(43.8)$ & $1360(52.2)$ & $1228(47.1)$ & $1144(43.9)$ & $840(32.3)$ \\
\hline \multicolumn{6}{|l|}{ Income (USD) } \\
\hline $0-<25000$ & $2611(25.0)$ & $782(30.0)$ & $710(27.2)$ & $641(24.6)$ & $478(18.4)$ \\
\hline $25000-<55000$ & $2948(28.3)$ & $821(31.5)$ & $747(28.7)$ & $753(28.9)$ & $627(24.1)$ \\
\hline$\geqslant 55000$ & 1165 (11.2) & $289(11.1)$ & $269(10.3)$ & $295(11.3)$ & $312(12.0)$ \\
\hline Unknown & $3704(35.5)$ & $716(27.5)$ & $881(33.8)$ & $919(35.2)$ & $1188(45.6)$ \\
\hline Physically active ${ }^{9}$ & 3275 (31.4) & $1016(39.0)$ & $890(34.1)$ & $755(29.0)$ & $614(23.6)$ \\
\hline \multicolumn{6}{|l|}{ Smoking status $^{+}$} \\
\hline Nonsmoker & $5066(48.6)$ & $973(37.3)$ & $1183(45.4)$ & $1385(53.1)$ & $1525(58.5)$ \\
\hline Former smoker & $2070(19.8)$ & $391(15.0)$ & $519(19.9)$ & $516(19.8)$ & $644(24.7)$ \\
\hline Current smoker & $3292(31.6)$ & $1244(47.7)$ & $905(34.7)$ & $707(27.1)$ & $436(16.7)$ \\
\hline \multicolumn{6}{|l|}{ Exam data } \\
\hline Height cm (mean \pm sD) & $168.2 \pm 10.0$ & $170.7 \pm 9.5$ & $168.4 \pm 10.1$ & $167.3 \pm 10.1$ & $166.3 \pm 9.7$ \\
\hline BMI $\mathrm{kg} \cdot \mathrm{m}^{-2}($ mean $\pm \mathrm{sD})$ & $29.03 \pm 6.82$ & $29.15 \pm 7.36$ & $29.42 \pm 6.80$ & $29.01 \pm 6.74$ & $28.53 \pm 6.31$ \\
\hline \multicolumn{6}{|l|}{ Classification } \\
\hline Underweight & $139(1.3)$ & $44(1.7)$ & $35(1.3)$ & $30(1.2)$ & $30(1.2)$ \\
\hline Normal & $2972(28.5)$ & $793(30.4)$ & $675(25.9)$ & $716(27.5)$ & $788(30.3)$ \\
\hline Overweight & $3430(32.9)$ & $815(31.3)$ & $849(32.6)$ & $891(34.2)$ & 875 (33.6) \\
\hline Obese & 3887 (37.3) & $956(36.7)$ & $1048(40.2)$ & $971(37.2)$ & $912(35.0)$ \\
\hline \multicolumn{6}{|l|}{ Questionnaire data } \\
\hline \multicolumn{6}{|l|}{ Asthma } \\
\hline Ever-asthma & $1501(14.4)$ & $420(16.1)$ & $382(14.7)$ & $367(14.1)$ & $332(12.7)$ \\
\hline Current asthma & $782(7.5)$ & $219(8.4)$ & $198(7.6)$ & $181(6.9)$ & $184(7.1)$ \\
\hline \multicolumn{6}{|l|}{ Bronchitis } \\
\hline Ever-bronchitis & $483(4.6)$ & $122(4.7)$ & $123(4.7)$ & $116(4.5)$ & $122(4.7)$ \\
\hline Current bronchitis & $205(2.0)$ & $65(2.5)$ & $52(2.0)$ & $41(1.6)$ & $47(1.8)$ \\
\hline \multicolumn{6}{|l|}{ Spirometry data ${ }^{\S, f}$} \\
\hline \multicolumn{6}{|c|}{ Pre-bronchodilator values (mean \pm SD) } \\
\hline $\mathrm{FEV}_{1} \mathrm{~L}$ & $3.2 \pm 0.9$ & $3.4 \pm 0.9$ & $3.2 \pm 0.9$ & $3.1 \pm 0.9$ & $3.0 \pm 0.8$ \\
\hline FVC L & $4.0 \pm 1.1$ & $4.3 \pm 1.0$ & $4.1 \pm 1.1$ & $3.9 \pm 1.0$ & $3.8 \pm 1.0$ \\
\hline $\mathrm{FEV}_{1} / \mathrm{FVC}$ ratio $\%$ & $79.0 \pm 7.7$ & $79.3 \pm 8.0$ & $79.6 \pm 7.5$ & $79.0 \pm 7.5$ & $78.3 \pm 7.5$ \\
\hline Per cent predicted valu & & & & & \\
\hline $\mathrm{FEV}_{1} \%$ & $98.4 \pm 15.3$ & $97.0 \pm 15.1$ & $98.5 \pm 14.9$ & $98.9 \pm 15.3$ & $99.3 \pm 15.6$ \\
\hline FVC \% & $102.0 \pm 14.2$ & $101.0 \pm 13.7$ & $101.9 \pm 14.0$ & $102.4 \pm 14.4$ & $102.8 \pm 14.7$ \\
\hline $\mathrm{FEV}_{1} / \mathrm{FVC}$ ratio $\%$ & $96.2 \pm 8.3$ & $95.7 \pm 8.6$ & $96.4 \pm 8.1$ & $96.3 \pm 8.1$ & $96.3 \pm 8.2$ \\
\hline Airway restriction & $304(1.6)$ & $91(3.5)$ & $78(3.0)$ & $76(2.9)$ & $59(2.3)$ \\
\hline COPD & $440(4.6)$ & $146(5.6)$ & $107(4.1)$ & $99(3.8)$ & $88(3.4)$ \\
\hline Total intake (mean \pm sD) & & & & & \\
\hline Calories & $2129 \pm 826$ & $2420 \pm 907$ & $2167 \pm 824$ & $2030 \pm 781$ & $1896 \pm 683$ \\
\hline Fat $\%$ & $32.7 \pm 7.6$ & $32.1 \pm 7.6$ & $32.4 \pm 7.2$ & $32.7 \pm 7.6$ & $33.6 \pm 8.0$ \\
\hline Protein \% & $16.1 \pm 4.3$ & $15.4 \pm 4.3$ & $15.7 \pm 4.1$ & $16.4 \pm 4.3$ & $16.8 \pm 4.3$ \\
\hline $\mathrm{CHO} \%$ & $49.8 \pm 9.7$ & $49.5 \pm 10.1$ & $50.4 \pm 9.4$ & $49.8 \pm 9.7$ & $49.6 \pm 9.6$ \\
\hline Fibre $g \cdot$ day $^{-1}$ & $16.9 \pm 9.0$ & $13.5 \pm 7.3$ & $15.6 \pm 8.1$ & $17.4 \pm 8.7$ & $21.2 \pm 10.1$ \\
\hline
\end{tabular}


TABLE 1 Continued

Quartile AHEI-2010 score\#

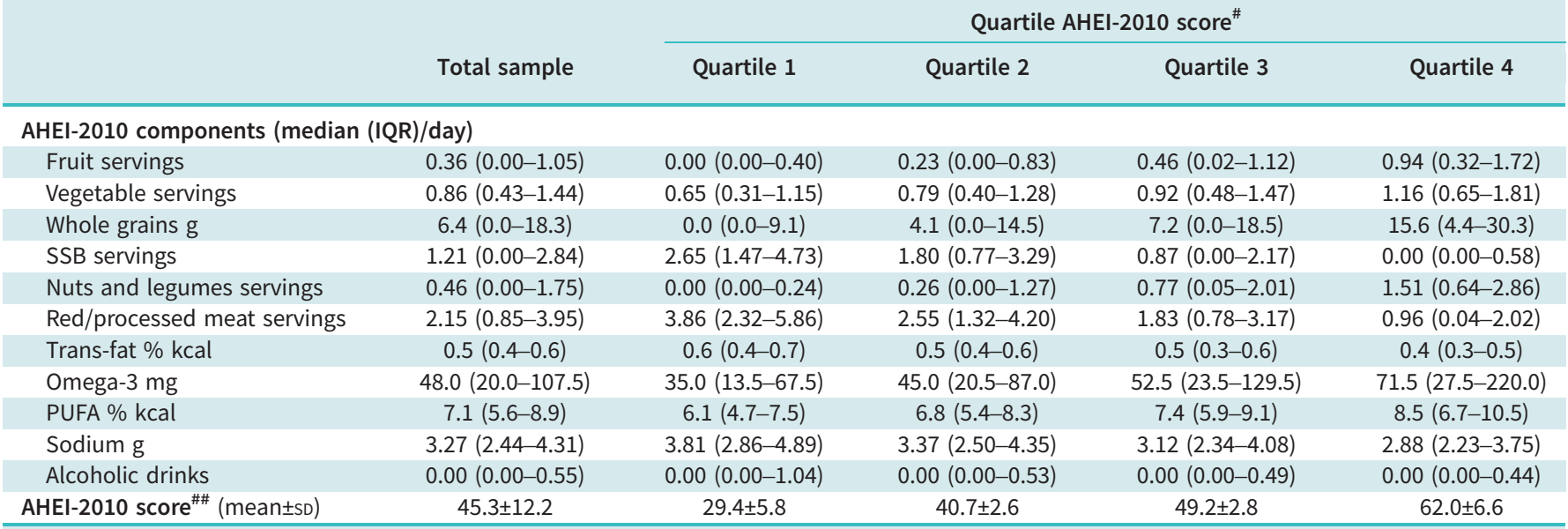

Data are presented as $\mathrm{n}(\%)$, unless otherwise indicated. BMI: body mass index; $\mathrm{FEV}_{1}$ : forced expiratory volume in $1 \mathrm{~s}$; FVC: forced vital capacity; SSB: sugar-sweetened beverages; PUFA: polyunsaturated fatty acids. ": differences in characteristics by quartile AHEI-2010 score were explored by Chi-square tests for categorical variables and one-way analysis of variance for continuous characteristics. All components except for spirometric restriction ( $\mathrm{p}$-value $=0.072$ ) were significantly different by quartile AHEI-2010 score ( $\mathrm{p}$-value $<0.001$ ). ${ }^{\top}$ : physical activity data: $n=10427$ (Missing 1). Physically active was defined as engaging in $150 \mathrm{~min}$ of moderate-intensity or $75 \mathrm{~min}$ of vigorous-intensity activity per week. ${ }^{+}$: current smokers reported never smoking or have urinary cotinine value $>10 \mathrm{ng} \cdot \mathrm{mL}^{-1}$. Former smokers reported they had quit smoking or have urinary cotinine value $1-10 \mathrm{ng} \cdot \mathrm{mL}^{-1}$. Never-smokers reported never smoking or have a urinary cotinine value $<1 \mathrm{ng} \cdot \mathrm{mL}^{-1}$. ${ }^{\S}$ : spirometry measures obtained in the entire sample $(n=10428)$. Lung function outcomes determined in participants with lung function measures less than the lower limit of normal (LLN). Spirometric restriction defined as FVC $<$ LLN. COPD defined as FEV $_{1} / F V C<L L N$. ${ }^{f}$ : NHANES sample weights were utilised to determine prevalence estimates of airway restriction and COPD. \#\#: AHEI-2010 score ranges from 0 to 110 (higher=better diet quality). Score increases with higher intakes of fruit, vegetable, whole grains, SSB, nuts and legumes, Omega-3, PUFA and moderate alcohol consumption. Score decreases with higher intakes of red/processed meat, trans-fat, sodium and excessive alcohol.

red/processed meat ( 2.15 servings $\cdot$ day $^{-1}$ versus 1.5 serving $\cdot$ day $^{-1}$ limit) and sodium $\left(3267 \mathrm{mg} \cdot \mathrm{day}^{-1}\right.$ versus $2300 \mathrm{mg} \cdot \mathrm{day}^{-1}$ limit) [26]. Utilising the 11-dietary components, the mean \pm SD AHEI-2010 score of participants was $45.3 \pm 12.2$ [17]. There were significant differences in all dietary components of participants by quartile diet quality score $(\mathrm{p}<0.001$ for all). Individuals with higher diet quality scores consumed more fruit, vegetables, whole grains, nuts, legumes, omega-3 fatty acids and polyunsaturated fatty acids than those with lower scores. Conversely, individuals with lower diet quality scores consumed more SSB, red/processed meat, trans-fat, sodium and alcohol than those with lower diet quality scores.

The association between diet quality and pre-bronchodilator lung function measures is shown in table 2 . Compared to those within the lowest quartile group, individuals in the second, third or fourth quartile had higher $\mathrm{FEV}_{1}$, FVC and $\mathrm{FEV}_{1} / \mathrm{FVC}$ ratio when controlling for age, sex and height (Model 1). After controlling for all additional confounders and accounting for the interaction of smoking and AHEI (Model 2), these associations remained statistically significant for $\mathrm{FEV}_{1}$ and FVC ( $\beta$-coefficient for the highest versus lowest quartile of AHEI 47.92, 95\% CI 2.27-93.57; and $\beta$-coefficient 80.23, 95\% CI 34.03-126.42, respectively). The adjusted associations of AHEI with $\mathrm{FEV}_{1}$ and FVC remained statistically significant when accounting for the interaction of ethnicity and AHEI (Model 3) on $\mathrm{FEV}_{1}$ ( $\beta$-coefficient for the highest versus lowest quartile of AHEI 95.52, 95\% CI 41.74-149.31) and on FVC ( $\beta$-coefficient for the highest versus lowest quartile of AHEI 119.49, 95\% CI 69.67-169.31). The adjusted subgroup analyses by race/ethnicity showed no evidence of association between diet quality and lung function in any of the race subgroups. Among never-smokers $(n=5066)$, former smokers $(n=2070)$ and current smokers $(n=3292)$, individuals in the highest quartile of AHEI had higher FVC compared to those in the lowest quartile group. There was no evidence of interaction between AHEI and ethnicity in this subgroup analysis.

The population-weighted-logistic regression models between diet quality and airway restriction $(\mathrm{FVC}<\mathrm{LLN})$, and COPD $\left(\mathrm{FEV}_{1} / \mathrm{FVC}<\mathrm{LLN}\right)$ are shown in table 3 . Compared to those in the lowest quartile group, individuals in the second, third or fourth quartile had lower odds of airway restriction and COPD when controlling for age, sex and height (Model 1). When controlling for additional covariates and for the interaction of smoking with AHEI (Model 2), this association was attenuated and was no longer statistically significant. When adjusting for all potential confounders and accounting for the interaction of race/ethnicity and AHEI (Model 3), those in the highest quartile of AHEI intake were less likely to have 
TABLE 2 Adjusted associations between quartile diet quality Alternative Healthy Eating Index (AHEI-2010) score and pre-bronchodilator lung function outcomes

Lung function measures ${ }^{\#}$

$\mathrm{FEV}_{1} \mathrm{~mL} \quad \mathrm{FVC} \mathrm{mL} \quad \mathrm{FEV}_{1} / \mathrm{FVC}$ ratio $\%$

\begin{tabular}{|c|c|c|c|}
\hline \multicolumn{4}{|l|}{ Total sample $n=10428$} \\
\hline \multicolumn{4}{|l|}{ Model $1^{\triangleleft}$} \\
\hline Quartile 1 (3.0-37.5) & Reference & Reference & Reference \\
\hline Quartile $2(35.5-46.0)$ & $91.3(45.4-137.3)$ & $82.3(35.7-128.9)$ & $0.81(0.15-1.47)$ \\
\hline Quartile 3 (43.7-55.4) & $102.2(63.1-141.2)$ & $106.7(58.6-154.7)$ & $0.64(0.09-1.19)$ \\
\hline Quartile 4 (52.5-94.6) & $167.7(122.5-212.9)$ & $180.7(131.2-230.1)$ & $0.77(0.06-1.49)$ \\
\hline \multicolumn{4}{|l|}{ Model $2^{+}$} \\
\hline Quartile 1 (3.0-37.5) & Reference & Reference & Reference \\
\hline Quartile 2 (35.5-46.0) & $14.82(-45.53-75.17)$ & $16.02(-45.37-77.40)$ & $0.19(-0.48-0.85)$ \\
\hline Quartile 3 (43.7-55.4) & $10.46(-35.91-56.83)$ & $33.96(-17.56-85.49)$ & $0.01(-0.69-0.71)$ \\
\hline Quartile 4 (52.5-94.6) & $47.92(2.27-93.57)$ & $80.23(34.03-126.42)$ & $-0.11(-0.79-0.58)$ \\
\hline Interaction of AHEI and smoking status ( $p$-value) & 0.532 & 0.231 & 0.585 \\
\hline \multicolumn{4}{|l|}{ Model $3^{\S}$} \\
\hline Quartile 1 (3.0-37.5) & Reference & Reference & Reference \\
\hline Quartile 2 (35.5-46.0) & $78.66(9.86-147.46)$ & $76.86(16.66-137.07)$ & $0.52(-0.22-1.26)$ \\
\hline Quartile 3 (43.7-55.4) & $63.17(10.13-116.21)$ & $80.31(23.27-137.35)$ & $0.07(-0.58-0.73)$ \\
\hline Quartile 4 (52.5-94.6) & $95.52(41.74-149.31)$ & $119.49(69.67-169.31)$ & $0.04(-0.74-0.83)$ \\
\hline Interaction of AHEI and race/ethnicity ( $p$-value) & 0.109 & 0.029 & 0.355 \\
\hline \multicolumn{4}{|l|}{ Subgroup analysis by race/ethnicity ${ }^{f, \# \#}$} \\
\hline Non-Hispanic white $(n=4452)$ & $64.43(2.09-126.78)$ & $106.94(48.24-165.64)$ & $-0.18(-1.15-0.79)$ \\
\hline Interaction of AHEI and smoking status ( $p$-value) & 0.484 & 0.107 & 0.496 \\
\hline Non-Hispanic black ( $\mathrm{n}=2185$ ) & $58.99(-25.91-143.89)$ & $69.88(-20.36-160.12)$ & $0.30(-1.24-1.84)$ \\
\hline Interaction of AHEI and smoking status ( $p$-value) & 0.586 & 0.568 & 0.258 \\
\hline Hispanic $(n=2918)$ & $11.05(-65.82-87.93)$ & $14.98(-70.38-100.33)$ & $-0.08(-0.87-0.71)$ \\
\hline Interaction of AHEI and smoking status ( $p$-value) & 0.011 & 0.306 & 0.039 \\
\hline \multicolumn{4}{|l|}{ Subgroup analysis by smoking status ${ }^{f, \uparrow ฯ ~}$} \\
\hline Never-smoker $(n=5066)$ & $19.03(-38.23-76.30)$ & $76.84(19.40-134.28)$ & $-0.99(-1.87--0.10)$ \\
\hline Interaction of AHEI and race/ethnicity ( $p$-value) & 0.872 & 0.730 & 0.236 \\
\hline Former smoker $(n=2070)$ & $85.60(-15.69-186.88)$ & $106.76(5.79-207.72)$ & $-0.05(-1.26-1.16)$ \\
\hline Interaction of AHEI and race/ethnicity ( $p$-value) & 0.174 & 0.276 & 0.477 \\
\hline Current smoker $(n=3292)$ & $160.18(54.41-265.95)$ & $165.72(61.31-270.13)$ & $0.93(-0.74-2.61)$ \\
\hline Interaction of AHEI and race/ethnicity ( $p$-value) & 0.117 & 0.491 & 0.322 \\
\hline
\end{tabular}

spirometric restriction (OR 0.23, 95\% CI 0.08-0.67). Adjusted subgroup analyses by race/ethnicity showed no evidence of association between AHEI and any of the three race/ethnicity subgroups. Subgroup analyses by smoking status showed no evidence of association between diet quality and spirometric restriction or COPD.

Higher quartile AHEI-2010 score remained statistically significantly associated with spirometric restriction when individually controlling for nuts/legumes, red/processed meat, omega-3 fatty acids, sodium and alcohol (supplementary Table S2). However, this association was attenuated to the null when individually controlling for fibre ( $p$-value $=0.17)$, fruit $(p$-value $=0.20)$, vegetables $(p$-value $=0.13)$, SSB $(p$-value=0.20), 


\begin{tabular}{|c|c|c|}
\hline & \multicolumn{2}{|c|}{ Lung function outcomes ${ }^{\#}$} \\
\hline & Airway restriction $(\mathrm{FVC}<\mathrm{LLN})$ & COPD $\left(\mathrm{FEV}_{1} / \mathrm{FVC}<\mathrm{LLN}\right)$ \\
\hline \multicolumn{3}{|l|}{ Total sample $(n=10428)$} \\
\hline \multicolumn{3}{|l|}{ Model $1^{4}$} \\
\hline Quartile 1 (3.0-37.5) & Reference & Reference \\
\hline Quartile $2(35.5-46.0)$ & $0.65(0.41-1.03)$ & $0.56(0.40-0.79)$ \\
\hline Quartile 3 (43.7-55.4) & $0.60(0.39-0.94)$ & $0.60(0.45-0.81)$ \\
\hline Quartile 4 (52.5-94.6) & $0.43(0.28-0.65)$ & $0.46(0.32-0.67)$ \\
\hline \multicolumn{3}{|l|}{ Model $2^{+}$} \\
\hline Quartile 1 (3.0-37.5) & Reference & Reference \\
\hline Quartile $2(35.5-46.0)$ & $0.81(0.39-1.71)$ & $0.52(0.22-1.24)$ \\
\hline Quartile 3 (43.7-55.4) & $0.64(0.30-1.35)$ & $0.93(0.40-2.17)$ \\
\hline Quartile 4 (52.5-94.6) & $0.67(0.35-1.26)$ & $0.73(0.35-1.51)$ \\
\hline Interaction AHEI and smoking status ( $p$-value) & 0.816 & 0.692 \\
\hline \multicolumn{3}{|l|}{ Model $3^{\S}$} \\
\hline Quartile 1 (3.0-37.5) & Reference & Reference \\
\hline Quartile 2 (35.5-46.0) & $0.38(0.13-1.08)$ & $0.60(0.40-0.91)$ \\
\hline Quartile 3 (43.7-55.4) & $0.44(0.18-1.07)$ & $0.81(0.57-1.15)$ \\
\hline Quartile 4 (52.5-94.6) & $0.23(0.08-0.67)$ & $0.69(0.46-1.02)$ \\
\hline Interaction AHEI and race/ethnicity (p-value) & 0.302 & 0.850 \\
\hline \multicolumn{3}{|l|}{ Subgroup analysis by race/ethnicity ${ }^{f, \# \#}$} \\
\hline Non-Hispanic white $(n=4452)$ & $0.43(0.05-3.73)$ & $0.85(0.31-2.35)$ \\
\hline Interaction AHEI and smoking status ( $p$-value) & Not-testable & 0.282 \\
\hline Non-Hispanic black ( $\mathrm{n}=2185)$ & $0.74(0.35-1.57)$ & $0.65(0.13-3.17)$ \\
\hline Interaction AHEI and smoking status ( $p$-value) & 0.370 & 0.222 \\
\hline Hispanic ( $n=2918$ ) & $0.41(0.06-2.85)$ & $0.75(0.13-4.27)$ \\
\hline Interaction AHEI and smoking status (p-value) & Not testable & Not testable \\
\hline \multicolumn{3}{|l|}{ Subgroup analysis by smoking status ${ }^{f, \uparrow ฯ}$} \\
\hline Never-smoker $(n=5066)$ & $0.62(0.08-5.12)$ & $0.85(0.29-2.48)$ \\
\hline Interaction AHEI and race/ethnicity ( $p$-value) & Not testable & 0.110 \\
\hline Former smoker $(\mathrm{n}=2070)$ & $1.81(0.12-27.9)$ & $0.96(0.41-2.23)$ \\
\hline Interaction AHEI and race/ethnicity ( $p$-value) & Not testable & Not testable \\
\hline Current smoker $(n=3292)$ & $0.37(0.09-1.49)$ & $0.64(0.32-1.31)$ \\
\hline Interaction AHEI and race/ethnicity (p-value) & Not testable & 0.692 \\
\hline \multicolumn{3}{|c|}{ 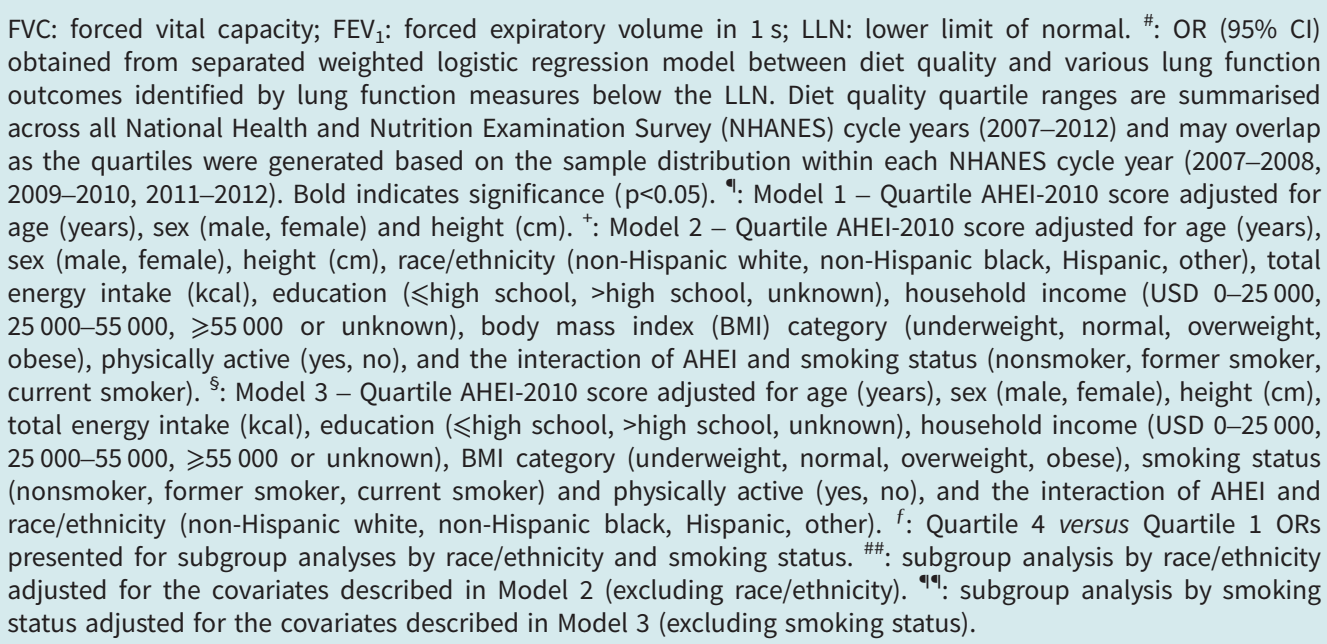 } \\
\hline
\end{tabular}

trans-fat ( $\mathrm{p}$-value=0.48) and PUFA ( $\mathrm{p}$-value=0.08). Individual adjustment with AHEI-2010 components did not modify the association between diet quality and COPD. Additional adjustment for dietary patterns are shown in supplementary Table S3. The association between AHEI with spirometric restriction or COPD remained statistically significant after adjustment by the fruit, fibre and legumes pattern. 
Discussion

In this population-based study representative of the general population of US adults, we found that diet quality, as measured by the AHEI-2010, was low. The mean \pm SD AHEI-2010 score was 45.3 \pm 12.2 , which is equivalent to meeting $41 \%$ of the daily recommendations to achieve an optimal diet quality (out of a total of 110) [19]. Higher diet quality (higher AHEI-2010) was positively and statistically significantly associated with higher $\mathrm{FEV}_{1}$ and FVC independent of a variety of relevant confounding factors [4, 29], and of interaction of AHEI and smoking habit, or AHEI and race/ethnicity on lung function outcomes. Adjusted subgroup analyses indicated the strongest associations in former or current smokers. To our knowledge, this is the first observational study of a nationally representative sample of US adults to report an association between AHEI-2010 score and lung function outcomes.

Evidence from observational studies has demonstrated that several components of diet considered to be healthy (e.g. fruits, antioxidants) have consistently been associated with better lung function $\left(\mathrm{FEV}_{1}, \mathrm{FVC}\right)$ in adults [9]. In European cohorts, higher intake of fruit was associated with slower lung function decline [7]. The beneficial properties of fruit include fibre and dietary flavonoids. Low fibre intake has been associated with reduced lung function measures in US adults [10]. Whereas higher intake of anthocyanin flavonoids, widely found in dark-pigmented fruits, has also been associated with slower lung function decline in a longitudinal analysis of older adults from the Veteran Affairs Normative Ageing Study in the US [30]. Beyond individual dietary components, other studies have associated dietary patterns with lung function measures. Dietary patterns considered prudent (rich in fruits, vegetables and oily fish) have been positively associated with $\mathrm{FEV}_{1}$ in a large cohort of adults from the UK participating in the Hertfordshire Cohort Study [31], whereas dietary patterns considered refined (high in fast food and snack foods) have been negatively associated with $\mathrm{FEV}_{1}$ and FVC in a national survey of Korean women [32].

In addition to lung function measures, higher diet quality was associated with decreased prevalence of spirometric restriction (restrictive lung disease) and trended towards reduced prevalence of COPD in this sample. Higher intake of dietary flavonoids has been associated with lower prevalence of spirometric restriction in a cohort study of European adults [33]. Evidence from this same cohort study did not identify significant associations between principal component analysis identified dietary patterns and COPD (FEV $/$ FVC $<$ LLN) [34], whereas other studies have demonstrated associations between dietary patterns and self-reported COPD. Dietary patterns considered prudent (rich in fruits, vegetables and oily fish) have been associated with decreased risk of self-reported COPD in a prospective study of US men [35]. In the Nurses' Health Study and the Health Professionals Follow-up Study, Varraso et al. [11] showed that higher AHEI-2010 scores were associated with decreased incidence of self-reported doctor-diagnosed COPD. These differences may be partly explained by the definitions used to ascertain COPD. In our study, we calculated airflow limitation (COPD) based on the $\mathrm{FEV}_{1} / \mathrm{FVC}$ LLN equations proposed by the American Thoracic Society and the European Respiratory Society, which are based on normal lung function seen in healthy lifetime non-smokers.

The mechanism between diet and lung function is hypothesised to relate to antioxidant properties of certain nutrients including vitamins $\mathrm{A}, \mathrm{C}$ and $\mathrm{E}, \beta$-carotene and omega-3 fatty acids [36-38]. These nutrients are mostly found in fruits and vegetables as well as in fatty fish [39], all foods that contribute to higher diet quality scores. Foods that contribute to lower diet quality scores, such as SSB, red/processed meat, trans-fat and sodium have all been associated with overweight/obesity [40-42], another risk factor for markers of poor lung function and prevalence of COPD [43, 44]. Intake of SSB was associated with increased risk of both airway restriction and COPD in this sample. In addition, diet quality was associated with spirometric restriction controlling for BMI classification suggesting that low-quality foods not only displace intake of higher quality antioxidant-containing foods but may also be contributing to inflammatory processes independent of weight status.

\section{Strengths}

AHEI-2010 is widely considered as a reliable indicator of the quality of diet of Americans and has been reported to be associated with several outcomes of non-communicable disease. Our study is the first to examine the association of AHEI-2010 and lung function in a nationally representative sample of US adults, using high-quality spirometry only, and calibrate the measures of pre-bronchodilator spirometry to improve the validity and reliability of the estimates of LLN. In addition, the analysis of data from NHANES offers a population-based estimate that is powered to detect associations, even controlling for a number of confounding factors. In terms of cigarette use, urine cotinine was used to identify current smoking status allowing for a more accurate assessment of this strongly associated factor with lung function beyond self-report. 


\section{Limitations}

Although the findings of this study contribute epidemiological evidence supporting the relationship between diet quality and lung function, it is only a cross-sectional analysis and therefore cannot establish temporality between the exposure and the outcome. In NHANES, dietary intake was ascertained with the use of a 24-hour recall questionnaire, which has some limitations, the most common of which is under-reporting of usual food intake, often explained by under-reporting of portion sizes consumed. To address these issues, the NHANES dietary survey was carried out using a multiple pass system. This system is known to improve reporting of dietary intake [45] by enquiring about intake in three main stages. First, participants are asked to name all the foods they consumed in the past day. Then they are asked more details about each food listed, including preparations used for their consumption. The final step is to enquire about portion sizes. The NHANES survey uses a set of measures specifically designed for the study's setting, with a target population of non-institutionalised civilians [13]. Highly trained dietary interviewers administer the 24-hour recall questionnaires, which ensured that as much relevant detail as possible was provided, further attenuating issues around misreporting of dietary intake. The 24-hour recall questionnaire is commonly used in national surveys, as it provides accurate estimates of mean intakes in specific groups (e.g. age, sex, ethnicity) in large population samples like that of NHANES [45]. However, the instrument has the additional limitation of providing information on dietary intake in a single day. In our study, $88 \%$ of participants contributed two 24-hour recall questionnaires, which helped to account for some variability in the diet. Despite this, we acknowledge that our estimates of diet quality might not reflect long-term dietary habits in the studied population.

\section{Conclusion}

This cross-sectional secondary analysis of adults aged 19-70 years in the US revealed that diet quality scores as measured by the AHEI-2010 were unsurprisingly low. Adults with higher diet quality scores had higher lung function measures and lower odds of restrictive lung disease, but not obstructive lung disease (COPD). These findings warrant replication in longitudinal studies to confirm whether a better diet quality can contribute to preserve lung function and slow down lung function decline in adults from the general population.

Provenance: Submitted article, peer reviewed.

Conflict of interest: None declared.

Support statement: This study was supported by: The Fulbright Commission and the Fundação para a Ciência e Tecnología (grant SFRH/BD/144563/2019); and the Ministerio de Ciência, Tecnología e Innovación (grant 860-2017). Funding information for this article has been deposited with the Crossref Funder Registry.

References

1 Murray CJL, Mokdad AH, Ballestros K, et al. The state of US health, 1990-2016: burden of diseases, injuries, and risk factors among US states. JAMA 2018; 319: 1444-1472.

2 Biener A, Decker SL, Rohde F. Prevalence and treatment of chronic obstructive pulmonary disease (COPD) in the United States. JAMA 2019; 322: 602.

3 Semega, J, Kollar M, Creamer J, et al. U.S. Census Bureau, Current Population Reports, P60-266(RV), Income and Poverty in the United States: 2018. Washington, DC, U.S. Government Printing Office, 2020.

4 Liu Y, Pleasants RA, Croft JB, et al. Smoking duration, respiratory symptoms, and COPD in adults aged $\geqslant 45$ years with a smoking history. Int J COPD 2015; 10: 1409-1416.

5 Kaluza J. Fruit and vegetable consumption and risk of COPD: a prospective cohort study of men. Thorax 2017; 72: 500-509.

6 Kaluza J, Harris HR, Linden A, et al. Long-term consumption of fruits and vegetables and risk of chronic obstructive pulmonary disease: a prospective cohort study of women. Int J Epidemiol 2018; 47: 1897-1909.

7 Garcia-Larsen V, Potts JF, Omenaas E, et al. Dietary antioxidants and 10-year lung function decline in adults from the ECRHS survey. Eur Respir J 2017; 50: 1602286.

8 Butland BK, Fehily AM, Elwood PC. Diet, lung function, and lung function decline in a cohort of 2512 middle aged men. Thorax 2000; 55: 102-108.

9 Scoditti E, Massaro M, Garbarino S, et al. Role of diet in chronic obstructive pulmonary disease prevention and treatment. Nutrients 2019; 11: 1357.

10 Hanson C, Lyden E, Rennard S, et al. The relationship between dietary fiber intake and lung function in the National Health and Nutrition Examination Surveys. Ann Am Thorac Soc 2016; 13: 643-650.

11 Varraso R, Chiuve SE, Fung TT, et al. Alternate Healthy Eating Index 2010 and risk of chronic obstructive pulmonary disease among US women and men: prospective study. BMJ 2015; 350: h286. 
12 CDC. National Health and Nutrition Examination Survey Data. 2012. www.cdc.gov/nchs/nhanes/about nhanes.htm Date last accessed: 2 September 2020.

13 CDC. National Health and Nutrition Examination Survey: 2011-2012 Data Documentation, Codebook, and Frequencies, Medical Conditions. 2012. wwwn.cdc.gov/nchs/nhanes/2011-2012/MCQ_G.htm Date last accessed: 2 September 2020.

14 CDC. National Health and Nutrition Examination Survey. Respiratory Health Spirometry Procedures Manual. Natl Heal Nutr Exam Surv 2008; 1-1-5-4. 2008. https://www.cdc.gov/nchs/data/nhanes/nhanes_07_08/ spirometry.pdf

15 Miller MR, Hankinson J, Brusasco V, et al. ATS/ERS Task Force: ATS/ERS Standardisation of Spirometry. Eur Respir J 2005; 26: 319-338.

16 Cooper BG, Stocks J, Hall GL, et al. The Global Lung Function Initiative (GLI) Network: bringing the world's respiratory reference values together. Breathe 2017; 13: e56-e64.

17 Quanjer PH, Stanojevic S, Cole TJ, et al. Multi-ethnic reference values for spirometry for the 3-95-yr age range: The global lung function 2012 equations. Eur Respir J 2012; 40: 1324-1343.

18 Kato B, Gulsvik A, Vollmer W, et al. Can spirometric norms be set using pre- or post- bronchodilator test results in older people? Respir Res 2012; 13: 102.

19 Chiuve SE, Fung TT, Rimm EB, et al. Alternative dietary indices both strongly predict risk of chronic disease. J Nutr 2012; 142: 1009-1018.

20 CDC. National Health and Nutrition Examination Survey: 2011-2012 Data Documentation, Codebook, and Frequencies, Demographic Variable and Sample Weights. 2012. wwwn.cdc.gov/Nchs/Nhanes/2011-2012/ DEMO_G.htm Date last accessed: 28 August 2020.

21 Benowitz NL, Perez-Stable EJ, Fong I, et al. Ethnic differences in N-glucuronidation of nicotine and cotinine. J Pharmacol Exp Ther 1999; 291: 1196-1203.

22 Hukkanen J, Jacob P III, Benowitz NL. Metabolism and disposition kinetics of nicotine. Pharmacol Rev 2005; 57: 79-115.

23 CDC. National Health and Nutrition Examination Survey: 2011-2012 Data Documentation, Codebook, and Frequencies, Physical Activity [Internet]. 2012. wwwn.cdc.gov/nchs/nhanes/2011-2012/PAQ_G.htm Date last accessed: 27 August 2020.

24 Centres for Disease Control and Prevention. Anthropometry procedures manual. https://www.cdc.gov/nchs/ data/nhanes/nhanes_07_08/manual_an.pdf

25 Black AE, Prentice AM, Goldberg GR, et al. Measurements of total energy expenditure provide insights into the validity of dietary measurements of energy intake. J Am Diet Assoc 1993; 93: 572-579.

26 DHHS. 2015-2020 Dietary Guidelines for Americans. 2015. https://health.gov/our-work/food-and-nutrition/ 2015-2020-dietary-guidelines/ Date last accessed: 27 August 2020.

27 Centers for Disease Control and Prevention. NHANES Tutorials - Module 3 - Weighting. 2020. wwwn.cdc.gov/ nchs/nhanes/tutorials/module3.aspx Date last accessed: 25 January 2021.

28 StataCorp. Stata Statistical Software: Release 14. College Station, StataCorp LP, 2015.

29 Mannino DM, Buist AS. Global burden of COPD: risk factors, prevalence, and future trends. Lancet 2007; 370: 765-773.

30 Mehta AJ, Cassidy A, Litonjua AA, et al. Dietary anthocyanin intake and age-related decline in lung function: longitudinal findings from the VA normative aging study. Am J Clin Nutr 2016; 103: 542-550.

31 Shaheen SO, Jameson KA, Syddall HE, et al. The relationship of dietary patterns with adult lung function and COPD. Eur Respir J 2010; 36: 277-284.

32 Cho Y, Chung HK, Kim SS, et al. Dietary patterns and pulmonary function in Korean women: findings from the Korea National Health and Nutrition Examination Survey 2007-2011. Food Chem Toxicol 2014; 74: 177-183.

33 Garcia-Larsen V, Thawer N, Charles D, et al. Dietary intake of flavonoids and ventilatory function in European adults: A GA²LEN study. Nutrients 2018; 10: 95.

34 Bakolis I, Hooper R, Bachert C, et al. Dietary patterns and respiratory health in adults from nine European countries: evidence from the GA²LEN study. Clin Exp Allergy 2018; 48: 1474-1482.

35 Varraso R, Fung TT, Hu FB, et al. Prospective study of dietary patterns and chronic obstructive pulmonary disease among US men. Thorax 2007; 62: 786-791.

36 Kelly $\mathrm{Y}$, Sacker A, Marmot M. Nutrition and respiratory health in adults: findings from the health survey for Scotland. Eur Respir J 2003; 23: 664-671.

37 Hu G, Cassano PA. Antioxidant Nutrients and Pulmonary Function: The Third National Health and Nutrition Examination Survey (NHANES III). Am J Epidemiol 2000; 151: 975-981.

38 Fiedor J, Burda K. Potential role of carotenoids as antioxidants in human health and disease. Nutrients 2014; 6: 466-488.

39 Liu RH. Health benefits of fruit and vegetables are from additive and synergistic combinations of phytochemicals. Am J Clin Nutr 2003; 78: Suppl. 3, 517S-520S. 
$40 \mathrm{Hu}$ FB, Malik VS. Sugar-sweetened beverages and risk of obesity and type 2 diabetes: epidemiologic evidence. Physiol Behav 2010; 100: 47-54.

41 Rouhani MH, Salehi-Abargouei A, Surkan PJ, et al. Is there a relationship between red or processed meat intake and obesity? A systematic review and meta-analysis of observational studies. Obes Rev 2014; 15: 740-748.

42 Grimes CA, Bolhuis DP, He FJ, et al. Dietary sodium intake and overweight and obesity in children and adults: a protocol for a systematic review and meta-analysis. Syst Rev 2016; 5: 7.

43 Dixon AE, Peters U. The effect of obesity on lung function. Expert Rev Respir Med 2018; 12: $755-767$.

44 Hanson C, Rutten EP, Wouters EFM, et al. Influence of diet and obesity on COPD development and outcomes. Int J COPD 2014; 9: 723-733.

45 Tucker KL. Assessment of usual dietary intake in population studies of gene-die interaction. Nutr Metab Cardiov Dis 2007; 17: 74-81. 